\title{
Tratamento Percutâneo da Insuficiência Mitral Secundária por MitraClip: Mitra-FR versus COAPT
}

\author{
Percutaneous Treatment of Secondary Mitral Regurgitation by MitraClip: Mitra-FR vs. COAPT
}

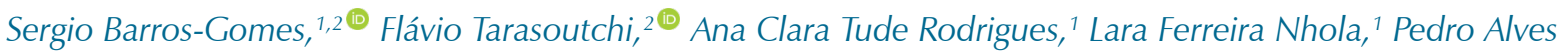 \\ Lemos, ${ }^{1}$ Samira Saady Morhy, ${ }^{1}$ Claudio Henrique Fischer, ${ }^{1,3}$ Marcelo Luiz Campos Vieira ${ }^{1,2}$ \\ Hospital Israelita Albert Einstein, ${ }^{2}$ São Paulo, SP - Brasil \\ Instituto do Coração da Universidade de São Paulo, ${ }^{1}$ São Paulo, SP - Brasil \\ Universidade Federal de São Paulo, ${ }^{3}$ São Paulo, SP - Brasil
}

\section{Introdução}

A insuficiência mitral (IM) secundária, ou funcional, deve-se a alterações na geometria do ventrículo esquerdo (VE) secundária à disfunção ventricular. ${ }^{1}$ Ocorre quando uma doença isquêmica cardíaca ou uma cardiopatia dilatada de qualquer etiologia causa dilatação do VE, dilatação do anel mitral e/ou deslocamento do músculo papilar, resultando em má coaptação das cúspides valvares e refluxo valvar. ${ }^{2}$ Dados estatísticos da American Heart Association indicam que 16.250 por milhão de americanos tenham IM secundária, ${ }^{3,4}$ números que totalizam mais de 5 milhões de casos somente nos EUA, e estima-se que esse número seja ainda maior tendo em vista o contínuo crescimento e envelhecimento da população. Isso é importante, uma vez que a IM secundária acarreta um mau prognóstico e é preditor independente de mortalidade. ${ }^{5,6}$

Por muitos anos, a intervenção mecânica da IM secundária (cirúrgica ou percutânea) foi restrita a casos refratários ao tratamento clínico convencional, 7,8 com evidência respaldada principalmente por dois importantes estudos do grupo Cardiothoracic Surgical Trials Network. 9,10 O primeiro estudo ${ }^{9}$ randomizou 301 pacientes com IM isquêmica de grau moderado e não encontrou diferenças na geometria ventricular entre os pacientes que receberam revascularização cirúrgica do miocárdico versus a combinação revascularização cirúrgica e reparo valvar mitral. O segundo estudo ${ }^{10}$ estudou 251 pacientes com IM grave e não encontrou diferenças com relação à mortalidade, além da maior recorrência de insuficiência mitral e taxas de complicações entre os pacientes tratados com plastia da válvula mitral versus troca valvar. Por causa desses dois estudos, as recomendações da American Heart Association/American College of Cardiology ${ }^{7}$ e a Diretriz Brasileira de Valvopatias ${ }^{8}$ classificaram a intervenção

\section{Palavras-chave}

Insuficiência Cardiaca; Insuficiência da Válvula Mitral; Ecocardiografia/métodos; Ensaios Clínicos.

\section{Correspondência: Sergio Barros-Gomes •}

Instituto do Coração da Universidade de São Paulo - Ecocardiograma - Av Dr. Eneas Carvalho de Aguiar, 44. CEP 05403-900, São Paulo, SP - Brasil E-mail: sergiomed2@hotmail.com

Artigo recebido em 26/01/2020, revisado em 16/10/2020, aceito em $02 / 12 / 2020$

DOI: https://doi.org/10.36660/abc.20200063 cirúrgica ou percutânea da válvula mitral como Classe IIb de indicação.

Até recentemente, nenhum estudo randomizado havia comparado a intervenção percutânea da IM secundária com o tratamento clínico convencional. Em 2018, a conduta frente à IM secundária mudou decisivamente com a apresentação de dois ensaios clínicos randomizados: o Multicentre Study of Percutaneous Mitral Valve Repair MitraClip Device in Patients with Severe Secondary Mitral Regurgitation (MITRA-FR) ${ }^{11}$ e o Cardiovascular Outcomes Assessment of the MitraClip Percutaneous Therapy for Heart Failure Patients with Functional Mitral Regurgitation (COAPT). ${ }^{12}$ Esses estudos avaliaram a eficácia e a segurança de duas estratégias terapêuticas em pacientes com IM secundária importante - a terapia percutânea com MitraClip ${ }^{\circledR}$ em conjunto com tratamento clínico otimizado versus tratamento clínico otimizado isolado.

Neste artigo, abordaremos as principais similaridades e diferenças entre os dois estudos e faremos considerações a respeito da aplicação desse procedimento na prática clínica, incluindo o perfil ideal do candidato para o procedimento (Tabela 1).

\section{MITRA-FR}

O MITRA-FR foi um estudo multicêntrico em 37 centros franceses que randomizou 304 pacientes com IM secundária importante, insuficiência cardíaca (IC) sistólica sintomática e fração de ejeção do ventrículo esquerdo (FEVE) entre 15\% e 40\% em duas estratégias terapêuticas, na razão de $1: 1$, alocados para tratamento percutâneo com MitraClip ${ }^{\circledR}$ em conjunto com tratamento clínico otimizado (grupo intervenção; 152 pacientes) versus tratamento clínico otimizado isolado (grupo controle; 152 pacientes). ${ }^{11}$ A IM secundária importante foi definida como tendo área do orifício regurgitante efetivo (ERO) $>20$ $\mathrm{mm}^{2}$ ou volume regurgitante (VR) $>30 \mathrm{~mL}$ por batimento. O desfecho primário foi mortalidade por qualquer causa ou hospitalização por IC dentro de 12 meses. Os pacientes dos dois grupos apresentaram uma melhora da classe funcional, mas sem diferença significativa entre os dois grupos. Por fim, não houve diferença significativa no desfecho primário combinado $(54,6 \%$ vs. $51,3 \%$, respectivamente; $p=0,53)$, taxa de mortalidade $(24,3 \%$ vs. $22,4 \% ; p>0,05)$ e taxa de hospitalização $(48,7 \%$ vs. $47,4 \%$; p >0,05) entre o grupo intervenção versus controle durante 1 ano de seguimento. Da mesma forma, não houve diferença significativa 


\begin{tabular}{|c|c|c|}
\hline Variável & MITRA-FR & COAPT \\
\hline Pacientes, $n$ & 304 & 614 \\
\hline $\begin{array}{l}\text { Pacientes intervenção/ } \\
\text { Controle, } \mathrm{n}\end{array}$ & $152 / 152$ & $302 / 312$ \\
\hline Tempo de estudo, anos & 2013-2017 & 2012-2017 \\
\hline \multicolumn{3}{|l|}{ Critério de inclusão } \\
\hline - ERO, mm² & $>20$ & $>30$ \\
\hline - VR, ml/batimento & 30 & 45 \\
\hline - FEVE, \% & $15-40$ & $20-50$ \\
\hline - DSFVE, mm & NA & $\leq 70$ \\
\hline - Medicações de base & Ajustadas em cada braço de acordo com a prática clínica & $\begin{array}{l}\text { Dose máxima estabilizada e terapia de ressincronização } \\
\text { se apropriado }\end{array}$ \\
\hline - Sintomas & NYHA II, III, IV & NYHA II, III, IV \\
\hline
\end{tabular}

DSFVE: diâmetro sistólico final do ventrículo esquerdo; ERO: orificio regurgitante efetivo; FEVE: fração de ejeção do ventrículo esquerdo; NYHA: New York Heart Association; VR: volume regurgitante.

no desfecho primário combinado $(63,8 \%$ vs. $67,1 \%$, respectivamente; $\mathrm{p}>0,05)$, taxa de mortalidade $(34,9 \%$ vs. $34,2 \% ; p>0,05)$ e taxa de hospitalização (55,9\% vs. 61,8\%; $\mathrm{p}>0,05$ ) entre o grupo intervenção versus controle durante 2 anos de seguimento. ${ }^{13}$ Os autores concluíram que o MitraClip ${ }^{\circledR}$ é seguro e efetivo na IM secundária em comparação ao tratamento clínico otimizado, mas sem melhora na sobrevida ou redução da hospitalização por IC em pacientes com IM secundária e IC sistólica.

\section{COAPT}

O COAPT foi um estudo multicêntrico que randomizou 614 pacientes em 78 centros americanos e canadenses com IC sistólica sintomática e IM secundária moderada a importante $(3+)$ ou importante $(4+)$, definida como ERO $>30 \mathrm{~mm}^{2}$ ou VR $>45 \mathrm{~mL}$ por batimento, com FEVE $\geq 20 \%$ (FEVE média $31,3 \pm 9,3 \%)$, na razão de $1: 1$, alocados para tratamento percutâneo com MitraClip ${ }^{\circledR}$ em conjunto com tratamento clínico otimizado (grupo intervenção; 302 pacientes) versus tratamento clínico otimizado isolado (grupo controle; 312 pacientes). ${ }^{12}$ IC sintomática foi definida como sintomas de IC apesar da dose máxima medicamentosa tolerada. O desfecho primário de eficácia foi hospitalização por IC dentro de 24 meses, e o desfecho primário de segurança foi evento livre de complicações relacionadas ao MitraClip ${ }^{\circledR}$ em 12 meses. A taxa anual de hospitalização por IC dentro de 24 meses foi 35,8\% por paciente/ano no grupo intervenção versus $67,9 \%$ no grupo controle (hazard ratio 0,53; 95\% IC 0,40-0,70; $p<0,001$ ). A porcentagem de evento-livre de complicações relacionadas ao dispositivo em 12 meses foi $96,6 \%$ ( $p<0,001$ ), enquanto morte por qualquer causa em 24 meses ocorreu em 29,1\% no grupo intervenção em comparação a $46,1 \%$ no grupo controle (hazard ratio 0,62; 95\% IC, 0,46-0,82; p <0,001). O grupo intervenção não somente reduziu a taxa de hospitalizações por IC em 47\%, mas também reduziu a mortalidade em 38\%. A redução no risco absoluto da mortalidade por todas as causas no grupo MitraClip ${ }^{\circledR}$ foi $17 \%$, e o número necessário para prevenir uma morte em 2 anos foi 5,9; já para prevenir uma hospitalização por IC em 2 anos foi 3,1. Os autores concluíram que a terapia combinada MitraClip ${ }^{\circledR}$ e tratamento clínico otimizado em pacientes com IC sistólica sintomática e IM moderada a importante ou importante reduz o número de hospitalizações por IC e a mortalidade por todas as causas em 2 anos, quando comparada com tratamento clínico otimizado exclusivamente. As Tabelas 2 e 3 comparam as características e os desfechos clínicos entre os dois estudos.

\section{Principais Similaridades e Diferenças}

Os dois ensaios tiveram resultados conflitantes, com o COAPT mostrando benefício do MitraClip ${ }^{\circledR}$ versus terapia medicamentosa, enquanto o MITRA-FR não mostrou benefício relacionado ao MitraClip ${ }^{\circledR}$. Não há dúvida de que esses dois estudos mudaram nosso entendimento de IM secundária. No entanto, por que eles apresentaram resultados significativamente diferentes? Por que o estudo COAPT teve um resultado positivo, enquanto o MITRA-FR foi neutro? Provavelmente as repostas a tais questões são multifatoriais e incluem diferenças na seleção de pacientes, otimização da terapia medicamentosa, grau da IM e no remodelamento ventricular.

Recrutamento: O recrutamento do COAPT foi mais seletivo em comparação ao MITRA-FR, visto que seu recrutamento foi mais lento e prolongado. O número de pacientes foi diferente nos dois estudos: COAPT recrutou cerca de 300 pacientes em cada braço, e o MITRA-FR cerca de 150 pacientes. Talvez o tamanho amostral da população do MITRA-FR, após exclusão de pacientes com seguimento incompleto, possa não ter sido suficiente para detectar significância estatística e, dessa forma, evitar o erro estatístico tipo II, principalmente em relação aos desfechos secundários. No estudo COAPT, o número de hospitalizações entre as duas estratégias terapêuticas divergiu desde o início do seguimento, parcialmente explicado pelo tratamento medicamentoso mais rigoroso.

Grau da IM: No MITRA-FR, a média de ERO foi $31 \mathrm{~mm}^{2}$, enquanto o COAPT teve média de ERO de $41 \mathrm{~mm} .{ }^{2}$ Apesar de o critério de inclusão de ambos os estudos ter sido IM ao 


\section{Ponto de Vista}

\begin{tabular}{|c|c|c|}
\hline Variável $^{\mathrm{a}}$ & MITRA-FR & COAPT \\
\hline \multicolumn{3}{|l|}{ Clínica } \\
\hline \multicolumn{3}{|l|}{ Idade, anos } \\
\hline - Braço MitraClip ${ }^{\circledR}$ & $70 \pm 10$ & $72 \pm 12$ \\
\hline - Braço controle & $71 \pm 10$ & $73 \pm 10$ \\
\hline \multirow{2}{*}{ Sexo, masculino, n (\%) } & $120(79)$ & $201(67)$ \\
\hline & $107(70)$ & (62) \\
\hline \multicolumn{3}{|l|}{ NYHA, \% } \\
\hline-1 & 0 & 0,2 \\
\hline$-\|$ & 32,9 & 39 \\
\hline- III & 58,5 & 52,5 \\
\hline$-I V$ & 8,6 & 8,3 \\
\hline \multicolumn{3}{|l|}{ Cardiomiopatia isquêmica } \\
\hline - Braço MitraClip® & $95(62,5)$ & $184(60,9)$ \\
\hline - Braço controle & $85(56,3)$ & $189(60,6)$ \\
\hline \multicolumn{3}{|l|}{ Revascularização coronariana prévia } \\
\hline - Braço MitraClip ${ }^{\circledR}$ & $71(46,7)$ & PCI: $130(43,0)$ CABG: $121(40,1)$ \\
\hline - Braço controle & $62(42,4)$ & PCI: $153(49,0)$ CABG: $126(40,4)$ \\
\hline \multicolumn{3}{|l|}{ Ressincronização cardíaca prévia } \\
\hline - Braço MitraClip ${ }^{\circledR}$ & $46(30,5)$ & $115(38,1)$ \\
\hline - Braço controle & $35(23,0)$ & $109(34,9)$ \\
\hline \multicolumn{3}{|l|}{ Risco Cirúrgico } \\
\hline - Escore STS & NA & $8,2 \pm 5,9 \%$ \\
\hline - EuroScore II & $6,2(3,5-11)$ & NA \\
\hline \multicolumn{3}{|l|}{ Ecocardiográfica } \\
\hline \multicolumn{3}{|l|}{ Gravidade da IM, \% } \\
\hline - ERO 20-29 mm² (moderada) & $157(52,2)$ & $80(13,5)$ \\
\hline - ERO 30-39 mm² (moderada/importante) & $95(31,6)$ & $270(45,7)$ \\
\hline - ERO $\geq 40 \mathrm{~mm}^{2}$ (importante) & $49(16,3)$ & $241(40,8)$ \\
\hline $\mathrm{ERO}, \mathrm{mm}^{2}$ & $31 \pm 10$ & $41 \pm 15$ \\
\hline VDFVEI, $\mathrm{mL} / \mathrm{m}^{2}$ & $135 \pm 35$ & $101 \pm 34$ \\
\hline FEVE, \% & $33 \pm 7$ & $31 \pm 9$ \\
\hline
\end{tabular}

menos moderada a importante, o estudo COAPT seguiu as recomendações americanas de 2008, ${ }^{14}$ que classifica como IM moderada a importante quando o ERO é $\geq 30 \mathrm{~mm}^{2}$ e/ ou VR de $45 \mathrm{~mL}$; já o MITRA-FR seguiu as recomendações europeias de 2012: ${ }^{15}$ ERO $\geq 20 \mathrm{~mm}^{2}$ e/ou VR de $30 \mathrm{~mL}$ como IM moderada a importante. Essa discordância é baseada no conceito de que a mortalidade em pacientes com IM secundária é significativamente maior para níveis menores de ERO e VR. ${ }^{16,17}$ Entretanto, o mecanismo da IM funcional é complexo e não se sabe se ERO ou VR moderados atuam ativamente como causadores do remodelamento e disfunção ventricular, ou se são meros marcadores resultantes da cardiomiopatia incipiente. As diretrizes subsequentes retornaram o ERO e o VR ao valor habitual; com base nas recomendações atuais, ERO de $30 \mathrm{~mm}^{2}$ é considerado moderado, enquanto ERO $\geq 40 \mathrm{~mm}^{2}$ é importante. ${ }^{8,18}$ Novos estudos sugerem que a abordagem unificada - baseada na integração de ERO, VR e fração regurgitante (FR) - possa ser um excelente discriminador de IM secundária importante quando comparada aos algoritmos estabelecidos nas últimas diretrizes e, portanto, um excelente identificador de pacientes com alto risco. ${ }^{19}$ Dito isso, um número significativo de pacientes (52\%) com IM moderada (ERO 20 a $30 \mathrm{~mm}^{2}$ ) foi recrutado no MITRA-FR, enquanto apenas $14 \%$ dos pacientes 
Ponto de Vista

\begin{tabular}{|c|c|c|}
\hline \multicolumn{3}{|l|}{ Tabela 3 - Desfecho clínico } \\
\hline Variávela & MITRA-FR & COAPT \\
\hline \multicolumn{3}{|l|}{ Grupo MitraClip ${ }^{\circledR}$ apenas, $n$} \\
\hline - Complicações no procedimento & $21(14,6)$ & $25(8,5)$ \\
\hline - IM $\geq+2$ na alta & $93(24,4)$ & $214(17,7)$ \\
\hline$-\mathrm{IM} \geq+2$ em 2 anos & $48(49,5)^{b}$ & $26(22,8)$ \\
\hline \multicolumn{3}{|c|}{ Mortalidade por qualquer causa em 2 anos, $n$} \\
\hline - Braço MitraClip ${ }^{\circledR}$ & $53(34,9)$ & $80(29,1)$ \\
\hline - Braço controle & $52(34,2)$ & $121(46,1)$ \\
\hline Valor $p$ & $>0,05$ & $<0,001$ \\
\hline \multicolumn{3}{|l|}{ Hospitalizações por ICC em 2 anos, n } \\
\hline - Braço MitraClip ${ }^{\circledR}$ & $85(55,9)$ & $92(35,7)$ \\
\hline - Braço controle & $94(61,8)$ & $151(56,7)$ \\
\hline Valor $p$ & $>0,05$ & $<0,001$ \\
\hline \multicolumn{3}{|c|}{$\begin{array}{l}\text { Mortalidade por qualquer causa } \\
\text { ou hospitalizações associadas a IC em } 2 \text { anos, n }\end{array}$} \\
\hline - Braço MitraClip ${ }^{\circledR}$ & $97(63,8)$ & $129(45,7)$ \\
\hline - Braço controle & $102(67,1)$ & $191(67,9)$ \\
\hline Valor $p$ & $>0,05$ & $<0,001$ \\
\hline
\end{tabular}

IC: insuficiência cardíaca; ICC: insuficiência cardiaca congestiva; IM: insuficiência mitral. a Variáveis categóricas são reportadas em números (porcentagens). ${ }^{b} I M \geq+2$ em 1 ano.

com essas características foram recrutados no COAPT. Já em relação à IM importante (ERO $\geq 40 \mathrm{~mm}^{2}$ ), apenas 16\% dos pacientes do MITRA-FR tinham IM importante versus $41 \%$ do COAPT. Os achados dos dois estudos sugerem que o benefício do MitraClip ${ }^{\circledR}$ é maior nos pacientes com ERO $>40 \mathrm{~mm}^{2}(i$. e., IM verdadeiramente importante).

Remodelamento Ventricular: O volume diastólico final do ventrículo esquerdo indexado (VDFVEI) médio dos pacientes do estudo MITRA-FR foi $135 \mathrm{~mL} / \mathrm{m}^{2}$ comparado a $101 \mathrm{~mL} / \mathrm{m}^{2}$ do COAPT. O VE foi significativamente maior no MITRA-FR, caracterizando ventrículos mais remodelados, em estágios mais avançados de cardiomiopatia. Essa diferença é provavelmente devido à exclusão de pacientes com dilatação/disfunção severa no COAPT (diâmetro sistólico do $V E>70 \mathrm{~mm}$ ), enquanto no MITRA-FR não havia essa limitação. O critério de inclusão da FEVE entre os dois estudos também foi diferente: o COAPT incluiu pacientes com FEVE $20 \%$ a $50 \%$ versus FEVE de $10 \%$ a $40 \%$ no MITRAFR. Interessantemente, um subgrupo de pacientes do estudo COAPT que não se beneficiou do tratamento por MitraClip ${ }^{\circledR}$ (número de hospitalizações associadas a IC em 12 meses) foi o dos pacientes com ERO e VDFVEI relativamente semelhantes àqueles recrutados no estudo MITRA-FR (ERO $\leq 30 \mathrm{~mm}^{2}$ e VDFVEI $\left.>96 \mathrm{~mL} / \mathrm{m}^{2}\right){ }^{20}$ Esses fatos sugerem que pacientes com IM moderada, VE marcadamente mais dilatados e com maior disfunção possam não ser os candidatos ideais para o tratamento com MitraClip ${ }^{\circledR}$. De fato, a alta recorrência de IM e o pior desfecho clínico já haviam sido reportados previamente na correção cirúrgica de pacientes com IM isquêmica, dilatação ventricular (diâmetro diastólico do VE >65 mm) e disfunção grave do
VE (FEVE <20\% e diâmetro sistólico do VE > 55 mm). ${ }^{21,22}$ No estudo MITRA-FR, a cardiomiopatia foi, possivelmente, o principal causador dos sintomas de IC e, consequentemente, o determinante do desfecho clínico desfavorável, ou seja, a IM foi meramente um fator secundário ao remodelamento ventricular. Em contrapartida, no COAPT, a IC foi, em parte, decorrente da IM e, por isso, o grau da IM no estudo COAPT foi maior, enquanto a cardiomiopatia foi menos avançada (VE de dimensão menor e FEVE maior).

Terapia Medicamentosa e Otimização Terapêutica: No estudo COAPT, o critério de inclusão de pacientes foi IC sistólica sintomática apesar da dose máxima medicamentosa tolerada, uso de terapia de ressincronização, uso de desfibriladores e terapia de revascularização cardíaca (se apropriado). Os pacientes foram otimizados clinicamente antes do recrutamento, e apenas poucos ajustes de medicação foram feitos no decorrer do seguimento. Já no MITRA-FR, não foi possível otimizar a medicação em todos os pacientes antes da randomização, e múltiplos reajustes ocorreram ao longo do seguimento. No MITRA-FR, a medicação foi ajustada pelos investigadores, enquanto no COAPT a medicação foi ajustada mais rigorosamente por um grupo de especialistas que supervisionavam a dose máxima tolerada, antes e após a intervenção. A posologia inicial e as doses ajustadas de cada medicação foram contabilizadas no estudo COAPT. Este rigor em termos de posologia e otimização medicamentosa implementada no estudo COAPT certamente não reflete a prática clínica diária.

Sucesso na Redução da IM: No final de 12 meses, $83 \%$ dos pacientes do MITRA-FR tiveram IM $\leq+2$ 
(moderada) em comparação a 95\% dos pacientes do COAPT. Consequentemente, $17 \%$ dos pacientes do MITRAFR tiveram IM $\geq+3$ (moderada/importante) em 12 meses em comparação a $5 \%$ dos pacientes do COAPT. O estudo COAPT teve uma estratégia mais agressiva em termos de clipes implantados quando comparado ao MITRA-FR (uso de um clipe em $36 \%$ dos casos para o COAPT vs. $46 \%$ para o MITRA-FR; dois clipes em 55\% dos casos do COAPT vs. 45\% para o MITRA-FR; três clipes em $8 \%$ dos pacientes do COAPT vs. 9\% do MITRA-FR; quatro clipes em 0,3\% dos pacientes do COAPT vs. $0 \%$ para o MITRA-FR). É possível que a maior taxa de sucesso na redução da IM possa estar associada a resultados favoráveis.

Fisiopatologia: Divergências em termos de fisiopatologia foram elegantemente demonstradas por Packer e Grayburn et al., ${ }^{23}$ que apresentaram o conceito de IM proporcional versus IM desproporcional com base na combinação de ERO e volume diastólico final (VDF) - razão ERO/VDF. Assumindo uma FEVE de $30 \%$ e uma fração regurgitante de $50 \%$ (perfil dos pacientes dos estudos), os autores mostraram graficamente que um ERO de $30 \mathrm{~mm}^{2}$ e um VDF maior (entre 220 e $240 \mathrm{~mL}$ ) poderia resultar em uma fração regurgitante de 50\%, assim como um ERO de $20 \mathrm{~mm}^{2}$ e um VDF normal poderiam resultar em uma fração regurgitante de $50 \% .{ }^{23}$ Os autores sugerem que o tratamento percutâneo da válvula mitral pelo MitraClip ${ }^{\circledR}$ seja mais benéfico em pacientes com IM desproporcional ao tamanho do VE isto é, quando a IM for maior do que a esperada para um VE dilatado, o tratamento com MitraClip ${ }^{\circledR}$ poderá ter um resultado mais favorável (ERO maior e VE menor). Em contrapartida, IM proporcional representaria pacientes mais doentes, com ventrículos maiores e menor grau de IM - em outras palavras, pacientes com cardiomiopatia em estágio avançado tardiamente selecionados para o tratamento intervencionista.

No entanto, Gaasch e Meyer et al., ${ }^{24}$ sugeriram que a gravidade da IM entre os dois estudos não seja tão diferente e que, de fato, seja semelhante. Os autores advogam que a fisiopatologia da IM é melhor descrita através do VR (ou a fração regurgitante) em vez do ERO. O VR é determinado pelo ERO e pela magnitude e duração do gradiente de pressão sistólica através da válvula regurgitante, ou seja, o ERO é apenas uma das variáveis determinantes do VR. É o VR que afeta o tamanho do VE a uma determinada FEVE, e exibe uma relação direta com o VDF. Desse modo, eles propuseram graficamente que a associação entre gravidade da IM e tamanho do VE seja baseada no conceito entre VR e VDF - razão VR/VDF -; o seu quociente é corrigido de maneira uniforme, tornando-o um índice adimensional. Assumindo uma fração regurgitante de $50 \%$ no estudo COAPT (suposição baseada na FEVE e em dados ecocardiográficos) e fração regurgitante de 53\% fornecida no MITRA-FR, a razão VR sobre VDF (VR/VDF) foi de 0,18 e 0,15, respectivamente. Esses coeficientes de proporcionalidade são relativamente baixos (ambos $<0,20)$ e semelhantes aos valores reportados em estudos prévios de IM secundária, refletindo uma contribuição proporcionalmente pequena do VR para um VDF grande. Assim, há um aumento desproporcional do VE no perfil dos pacientes dos dois estudos tipicamente visto em pacientes com IM secundária (IM desproporcional) em comparação aos pacientes com IM primária (VDF proporcional ao VR).
Volumes subestimados: No estudo COAPT, os pacientes tiveram um ERO médio de $41 \pm 15 \mathrm{~mm}^{2}$, o que corresponde a um VR de no mínimo 45 a $60 \mathrm{~mL}$. O volume ejetado total do VE no estudo COAPT foi $57 \mathrm{~mL}$ (volume diastólico final do VE subtraído pelo volume sistólico final), o que é totalmente incompatível para manter um débito cardíaco satisfatório. Assumindo um volume ejetado total do VE de $57 \mathrm{~mL}$, então o VR é o volume ejetado total do VE subtraído pelo volume ejetado para a via de saída (ou seja, o volume ejetado total do VE é igual ao VR mitral mais o volume ejetado na via de saída). Com base nesses cálculos, o volume ejetado para a frente é de 0 a 15 $\mathrm{mL}$, o que seria incompatível com a vida. Está claro que o VDF no estudo COAPT está subestimado. Se assumirmos um ERO de $41 \mathrm{~mm}^{2}$ e um VR de $60 \mathrm{~mL}$ (aproximado do estudo COAPT), então o VDF deveria ser maior que $300 \mathrm{~mL}$ (assumindo uma fração regurgitante de 50\% e FEVE de 31\% como relatado no estudo). De qualquer forma, o diâmetro diastólico do VE foi menor no estudo COAPT (média de $69 \mathrm{~mm}$ no MITRA-FR vs. $62 \mathrm{~mm}$ no COAPT) confirmando VE menores.

De fato, a quantificação da IM secundária através do ecocardiograma bidimensional é desafiadora devido a inúmeras limitações do próprio método, além da complexa fisiopatologia da IM. Em pacientes com IM funcional, em sua grande maioria, o ERO e o VR pelo método PISA são subestimados com os valores da ressonância cardíaca ${ }^{25}$ e ecocardiografia tridimensional. ${ }^{26}$ O orifício não circular e o comportamento dinâmico da IM contribuem significativamente para essas diferenças. Talvez a fração regurgitante possa suprir essas limitações e corroborar como variável essencial de gravidade, além do seu importante papel prognóstico. ${ }^{12} \mathrm{~A}$ fração regurgitante é calculada pela razão do VR sobre o volume ejetado total (VR/volume ejetado total), que, apesar de serem variáveis dependentes de condições de carga, tamanho e função do VE, o seu quociente é corrigido de maneira uniforme por esses parâmetros, podendo assim ser um indicador mais robusto. ${ }^{27}$

Outros fatores: Vale lembrar que, diferentemente da IM primária, em que sua gravidade é puramente quantificada com base no grau da IM, a IM secundária é complexa, heterogênea e influenciada por vários fatores: idade, doença de base subjacente, comorbidades, remodelamento do VE, extensão do infarto, distúrbios hemodinâmicos, entre outros. ${ }^{28}$ No estudo COAPT, o desfecho combinado mortalidade ou internação por insuficiência cardíaca no braço que recebeu terapia por MitraClip ${ }^{\circledR}$ foi relativamente alto (46\%). Isso mostra que, independentemente do reparo da válvula, esses pacientes continuam tendo um prognóstico reservado, visto que boa parte do risco é relacionada a esses fatores.

Nessa mesma linha, Cavalcante et al. ${ }^{29}$ revelaram que a fração regurgitante e o tamanho do infarto medidos em pacientes com cardiopatia isquêmica é um importante estratificador de risco que vai além do tamanho do VE e outras variáveis clínicas. Os autores também mostraram que o prognóstico desses pacientes é pior à medida que o tamanho do infarto e do grau de IM aumentam. Interessantemente, a extensão da fibrose não foi medida nos estudos MITRA-FR e COAPT, mas acreditamos que certamente teve impacto clínico no desfecho desses estudos. Talvez, pacientes com um coração maior e com uma maior área de infarto não possam se beneficiar do MitraClip ${ }^{\circledR}$; do mesmo modo, podemos especular que os pacientes do 
estudo MITRA-FR continham maior área de fibrose e, por isso, menor benefício com terapia por MitraClip ${ }^{\circledR}$. Novos estudos correlacionando desfechos clínicos em pacientes tratados por MitraClip ${ }^{\circledR}$ e extensão da fibrose seriam interessantes.

\section{Implicações na Prática Clínica}

Os dois estudos avaliaram a mesma entidade clínica: a IM funcional ou secundária. No COAPT, os pacientes eram sintomáticos (apesar da rigorosa terapia clínica otimizada), tinham IM mais importante, VE menores e melhor função sistólica em relação ao MITRA-FR. No estudo MITRA-FR, os pacientes apresentavam IM menos importante, VE maiores e com pior função sistólica, em estágio mais avançado de cardiomiopatia. A disfunção ventricular foi o principal causador da IC e de desfechos clínicos e, por isso, a terapia com MitraClip ${ }^{\circledR}$ pode não ser tão benéfica. ${ }^{30}$

A identificação antecipada da IM secundária antes que o VE se dilate em excesso é crucial. Embora seja considerada sucesso de procedimento IM residual $\leq+2$ (moderada), a meta do procedimento deve ser IM $\leq+1$ (leve) e a implantação de clipes adicionais deve ser considerada com o objetivo de atingir essa meta. Tendo em vista os achados do COAPT e MITRA-FR, acreditamos que os dois estudos são complementares. Esperamos que o estudo randomizado RESHAPE-HF (A Randomized Study of the MitraClip Device in Heart Failure Patients with Clinically Significant Functional Mitral Regurgitation), ${ }^{31}$ ainda em recrutamento e com o mesmo critério de inclusão do COAPT, possa fornecer um entendimento ainda maior na fisiopatologia da IM secundária, especialmente após dados conflitantes.

Ainda estamos no processo de definição do candidato ideal para o tratamento da insuficiência mitral secundária por MitraClip ${ }^{\circledR}$. É possível que o tamanho do infarto e/ou fibrose também possam ajudar na melhor seleção desses pacientes. ${ }^{31,32}$
Além disso, é necessário certificar que a gravidade da IM é puramente atribuível à gravidade da IM e não a outros fatores de risco e fatores confundidores. O estudo COAPT reforça o importante papel da IM na fisiopatologia da IC sistólica e, com a seleção apropriada de pacientes - excluindo os VE maiores, mais doentes, com maior área de fibrose e IM moderada e selecionando pacientes nos quais a IM seja tão severa que contribua para a gravidade da doença -, o tratamento percutâneo da IM secundária por MitraClip ${ }^{\circledR}$ pode ser benéfico, desde que obedeça aos seguintes critérios (Figura 1):

(1) Garantia de que a gravidade da IM seja puramente atribuível à gravidade da IM e não a outros fatores que influenciam a IM (idade, comorbidades, outras doenças cardíacas, grau de disfunção ventricular, extensão da fibrose, extensão do remodelamento).

(2) Avaliação da gravidade da IM pela integração de múltiplos parâmetros além do ERO: VR, fração regurgitante e possível quantificação da extensão da área de fibrose.

(3) IM $\geq+3$ (moderada a importante), definida como ERO $\geq 30 \mathrm{~mm}^{2} \mathrm{e} /$ ou VR de $45 \mathrm{~mL}$ por batimento.

(4) FEVE de $20 \%$ a 50\% e diâmetro sistólico do VE $<70 \mathrm{~mm}$.

(5) Sintomas de IC, apesar da terapia clínica otimizada (máxima dose tolerada), incluindo terapia de ressincronização e revascularização coronariana, se apropriada.

(6) Grupo intervencionista com experiência, com sucesso técnico na redução da $I M \geq+2$ maior que $95 \%$.

(7) Presença de um time multidisciplinar (heart team) para manejo, tratamento e otimização da IC.

(8) Após intervenção, acompanhamento de perto das medicações e do status volêmico.

(9) Identificação antecipada da IM secundária e encaminhamento a um time multidisciplinar (heart team) antes

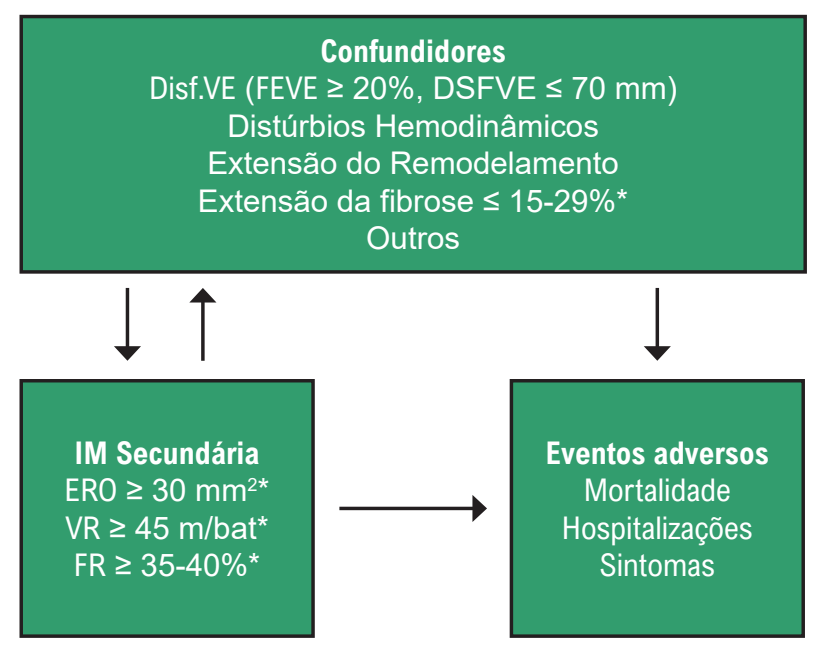

Adaptado de Marwick TH et al. JACC Cardiovsc Imaging. 2014 Mar; 7(3):333-5.

Figura 1 - A complexidade da insuficiência mitral funcional e a seleção do candidato ideal* para o implante de MitraClip®. Disf.: disfunção; DSFVE: diâmetro sistólico final do ventrículo esquerdo; ERO: orifício regurgitante efetivo; FEVE: fração de ejeção do ventrículo esquerdo; FR: fração regurgitante; $V R$ : volume regurgitante. 
que o ventrículo dilate muito ou o paciente seja hospitalizado, necessitando de cuidados intensivos ou de suporte inotrópico.

\section{Contribuição dos autores}

Concepção e desenho da pesquisa: Barros-Gomes S; Redação do manuscrito: Barros-Gomes S, Lemos PA, Fischer $\mathrm{CH}$, Vieira MLC; Revisão crítica do manuscrito quanto ao conteúdo intelectual importante: Barros-Gomes S, Tarasoutchi F, Rodrigues ACT, Nhola LF, Lemos PA, Morhy SS.

\section{Potencial conflito de interesse}

Não há conflito com o presente artigo

\section{Referências}

1. Asgar AW, Mack MJ, Stone GW. Secondary mitral regurgitation in heart failure: pathophysiology, prognosis, and therapeutic considerations. J Am Coll Cardiol. 2015 Mar 31;65(12):1231-1248. doi: 10.1016/j.jacc.2015.02.009.

2. Yiu SF, Enriquez-Sarano M, Tribouilloy C, Seward JB, Tajik AJ. Determinants of the degree of functional mitral regurgitation in patients with systolic left ventricular dysfunction: A quantitative clinical study. Circulation. 2000 Sep 19;102(12):1400-6.

3. de Marchena E, Badiye A, Robalino G, Junttila J, Atapattu S, Nakamura M, etal. Respective prevalenceof the different carpentier classes of mitral regurgitation: a stepping stone for future therapeutic research and development. J Card Surg. 2011 Jul;26(4):385-92. doi: 10.1111/j.1540-8191.2011.01274.x.

4. Benjamin EJ, Virani SS, Callaway CW, Chamberlain AM, Chang AR, Cheng S, et al. Heart Disease and Stroke Statistics-2018 Update: A Report From the American Heart Association. Circulation. 2018 Mar 20;137(12):e67-e492.

5. Goliasch G, Bartko PE, Pavo N, Neuhold S, Wurm R, Mascherbauer J, et al. Refining the prognostic impact of functional mitral regurgitation in chronic heart failure. Eur Heart J. 2018 Jan 1;39(1):39-46. doi: 10.1093/eurheartj/ ehx402.

6. Rossi A, Dini FL, Faggiano P, Agricola E, Cicoira M, Frattini S, etal. Independent prognostic value of functional mitral regurgitation in patients with heart failure. A quantitative analysis of 1256 patients with ischaemic and non-ischaemic dilated cardiomyopathy. Heart. 2011 Oct;97(20):1675-80. doi: 10.1136/ hrt.2011.225789.

7. Nishimura RA, Otto CM, Bonow RO, Carabello BA, Erwin JP, O'Gara PT, et al. $2014 \mathrm{AHA} / \mathrm{ACC}$ guideline for the management of patients with valvular heart disease: a report of the American College of Cardiology/American Heart Association Task Force on Practice Guidelines. J Thorac Cardiovasc Surg. 2014 Jul;148(1):e1-e132. doi: 10.1016/j.jtcvs.2014.05.014

8. Tarasoutchi F, Montera MW, Ramos Al de O, Sampaio RO, Rosa VEE, Accorsi TAD, et al. Atualização das Diretrizes Brasileiras de Valvopatias: Abordagem das Lesões Anatomicamente Importantes. Arq Bras Cardiol. 2017; 109(6 suppl 2):1-34. doi: 10.5935/abc.20180007.

9. Michler RE, Smith PK, Parides MK, Ailawadi G, Thourani V, Moskowitz AJ, et al. Two-Year Outcomes of Surgical Treatment of Moderate Ischemic Mitral Regurgitation. N Eng/ J Med. 2016 May 19;374(20):1932-41. doi: 10.1056/ NEJMoa 1602003.

10. Goldstein D, Moskowitz AJ, Gelijns AC, Ailawadi G, Parides MK, Perrault LP, et al. Two-Year Outcomes of Surgical Treatment of Severe Ischemic Mitral Regurgitation. N Engl J Med. 2016 Jan 28;374(4):344-53. doi: 10.1056/ NEJMoa1512913.

11. Obadia J-F, Messika-Zeitoun D, Leurent G, lung B, Bonnet G, Piriou N, et al. Percutaneous Repair or Medical Treatment for Secondary Mitral Regurgitation. NEngl/ Med. 2018 Dec 13;379(24):2297-2306. doi: 10.1056/ NEJMoa1805374.

\section{Fontes de financiamento}

O presente estudo não teve fontes de financiamento externas.

\section{Vinculação acadêmica}

Não há vinculação deste estudo a programas de pósgraduação.

\section{Aprovação ética e consentimento informado}

Este artigo não contém estudos com humanos ou animais realizados por nenhum dos autores.
12. Stone GW, Lindenfeld J, Abraham WT, Kar S, Lim DS, Mishell JM, et al. Transcatheter Mitral-Valve Repair in Patients with Heart Failure. N Engl J Med. 2019 May 16;380(20):1980-1981. doi: 10.1056/NEJMc1903982.

13. Iung B, Armoiry X, Vahanian A, Boutitie F, Mewton N, Trochu JN, et al. Percutaneous repair or medical treatment for secondary mitral regurgitation: outcomes at 2 years. Eur J Heart Fail. 2019 Dec;21(12):1619-1627. doi: 10.1002/ejhf.1616.

14. Bonow RO, Chatterjee K, de Leon AC, Faxon DP, Gaasch WH, O'Rourke RA, et al. 2008 focused update incorporated into the ACC/AHA 2006 guidelines for the management of patients with valvular heart disease: a report of the American College of Cardiology/American Heart Association Task Force on Practice Guidelines (Writing Committee to revise the 1998 guidelines for the management of patients with valvular heart disease). Endorsed by the Society of Cardiovascular Anesthesiologists, Society for Cardiovascular Angiography and Interventions, and Society of Thoracic Surgeons. J Am Coll Cardiol. 2008 Sep 23;52(13):e1-142. doi: 10.1016/j.jacc.2008.05.007.

15. Joint Task Force on the Management of Valvular Heart Disease of the European Society of Cardiology (ESC), European Association for CardioThoracic Surgery (EACTS), Vahanian A, Alfieri O, Andreotti F, Antunes MJ, et al. Guidelines on the management of valvular heart disease (version 2012). EurJ Cardiothorac Surg. 2012 Oct;42(4):S1-44. doi: 10.1093/ejcts/ezs455.

16. Grigioni F, Détaint D, Avierinos J-F, Scott C, Tajik J, Enriquez-Sarano M. Contribution of ischemic mitral regurgitation to congestive heart failure after myocardial infarction. J Am Coll Cardiol. 2005 Jan 18;45(2):260-7.

17. Grigioni F, Enriquez-Sarano M, Zehr KJ, Bailey KR, Tajik AJ. Ischemic mitral regurgitation: long-term outcome and prognostic implications with quantitative Doppler assessment. Circulation.2001;103(13):1759-64

18. Nishimura RA, Otto CM, Bonow RO, Carabello BA, Erwin JP, Fleisher LA, et al. 2017 AHA/ACC Focused Update of the 2014 AHA/ACC Guideline for the Management of Patients With Valvular Heart Disease: A Report of the American College of Cardiology/American Heart Association Task Force on Clinical Practice Guidelines. J Am Coll Cardiol. 2017;135(25):2451-577.

19. Bartko PE, Arfsten H, Heitzinger G, Pavo N, Toma A, Strunk G, et al. A Unifying Concept for the Quantitative Assessment of Secondary Mitral Regurgitation. J Am Coll Cardiol. 2019 May 28;73(20):2506-17

20. Stone GW. Pivotal transcatheter FMR device trials: focus on COAPT and MITRA-FR, with implications for other transcatheter mitral valve device investigations. [Cited in 2019 Jan 23]. Available from: https://www.tctmd. $\mathrm{com} /$ slide/pivotal-transcatheter-fmr-device-trials-focus- coapt-and-mitrafr-implications-other.

21. Braun J, Bax JJ, Versteegh MIM, Voigt PG, Holman ER, Klautz RJM, et al. Preoperative left ventricular dimensions predict reverse remodeling following restrictive mitral annuloplasty in ischemic mitral regurgitation. Eur J Cardiothorac Surg. 2005 May;27(5):847-53. 
22. Braun J, van de Veire NR, Klautz RJM, Versteegh MIM, Holman ER, Westenberg JJM, et al. Restrictive mitral annuloplasty cures ischemic mitral regurgitation and heart failure. Ann Thorac Surg. 2008 Feb;85(2):430-6; discussion 436-7. doi: 10.1016/j.athoracsur.2007.08.040.

23. Grayburn PA, Cardiovascular ASJ, 2019. Proportionate and disproportionate functional mitral regurgitation: a new conceptual framework that reconciles the results of the MITRA-FR and COAPT trials. JACC Cardiovasc Imaging. 2019 Feb;12(2):353-62. doi: 10.1016/j. jcmg.2018.11.006

24. Gaasch WH, Aurigemma GP, Meyer TE. An appraisal of the association of clinical outcomes with the severity of regurgitant volume relative to end-diastolic volume in patients with secondary mitral regurgitation. JAMA Cardiol. 2020;5(4):476-81.

25. Uretsky S, Aldaia L, Marcoff L, Koulagiannis K, Hiramatsu S, Angulian E, et al. The effect of systolic variation of mitral regurgitation on discordance between noninvasive imaging modalities. JACC CardiovasC Imaging. 2019;12(12): 2431-42. doi:10.1016/j.jcmg.2019.02.014

26. Matsumura Y, Fukuda S, Tran H, Greenberg NL, Agler DA, Wada $\mathrm{N}$, et al.Geometry of the proximal isovelocity surface area in mitral regurgitation by 3-dimensional color Doppler echocardiography: difference between functional mitral regurgitation and prolapse regurgitation. Am Heart J. 2008;155(2):231-8. doi:10.1016/j.ahj. 2007.09.002

27. Hahn RT. Disproportionate Emphasis on Proportionate Mitral RegurgitationAre There Better Measures of Regurgitant Severity? JAMA Cardiol 2020 Feb 19. doi: 10.1001/jamacardio.2019.6235.

28. Marwick TH, Lancellotti P, Pierard L. Ischaemic mitral regurgitation: mechanisms and diagnosis. Heart 2009;95:1711-8.

29. Cavalcante JL, Kusnose K, Obuchowski NA, et al. Prognostic impact of ischemic mitral regur- gitation severity and myocardial infarct quantifi-cation by cardiovascular magnetic resonance. J Am Coll Cardiol Img 2020;13:1489501.

30. Pibarot P, Delgado V, Bax JJ. MITRA-FR vs. COAPT: lessons from two trials with diametrically opposed results. Eur Heart J Cardiovasc Imaging. 2019 Jun 1;20(6):620-4. doi: 10.1093/ehjci/jez073.

31. A Randomized Study of the MitraClip Device in Heart Failure Patients With Clinically Significant Functional Mitral Regurgitation (RESHAPE-HF) (ClinicalTrials.gov website). 2019. Available at: $h$ ttps://clinicaltrials.gov/ct2/ show/NCT01772108. Accessed February 15, 2019.

32. Carabello BA, Boyd WD. Scar in Secondary MR, Another Piece to the Puzzle: Dead Meat Don't Beat. JACC Cardiovasc Imaging 2020 Jul;13(7):1502-4. 